\title{
Novel Techniques for Microspectroscopy and Chemical Imaging Analysis of Soybean Seeds and Embryos
}

I.C. Baianu*,+, Tiefeng You*, Doina Costescu+

*Department of Food Science \& Human Nutrition

+Department of Nuclear, Plasma, and Radiological Engineering

University of Illinois at Urbana-Champaign 


\section{Abstract}

$\square$ Novel methodologies are currently being evaluated for the Chemical Analysis of soybean seeds as well as developing and mature soybean embryos by FT-IR/NIR Microspectroscopy.

$\square$ This is the first report of FT-NIR Chemical Images obtained with 1 micron resolution. NIR spectra of Proteins, Oil and DNA fibers are obtained for regions as small as $1 \mu^{2}$. 


\section{Instruments and Samples}

Four commercial IR/NIR instruments available in 2002 from PerkinElmer Co. (Shelton, CT, USA) were employed in this study:

$>$ FT-NIR Spectrometer (SpectrumOne-NTS)

$>$ FT-IR Spectrometer (SpectrumOne)

$>$ FT-NIR Microscope (NIR AutoImage)

$>$ FT-IR Microscope (Spotlight300)

Intact Soybean seeds, soybean Embryos, and standard test samples were investigated in the spectral range from 11,000 to $700 \mathrm{~cm}^{-1}$, with spectral resolutions to $0.5 \mathrm{~cm}^{-1}$. Chemical Images were obtained both in the Reflection and Transmission Mode in as little as 3 minutes (at $8 \mathrm{~cm}^{-1}$ resolution). The best microscope resolution of $\sim 1$ micron was obtained with the NIR AutoImage Microscope coupled to the SpectrumOne-NTS spectrometer. 


\section{FT-IR Spectrometer Spectrum One and FT-NIR Spectrometer SpectrumOne-NTS}

$\square$ Introduced in 2001 by Perkin Elmer Co. (Shelton, CT, USA) for High Sensitivity, high-resolution and long-term Stability

$\square$ Spectrum One and Spectrum One NTS have a similar look but are configured for different spectral ranges.

$\square$ Both instruments were employed

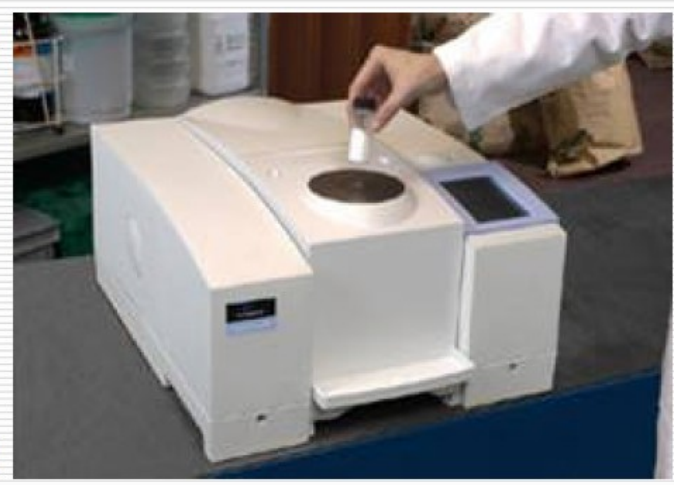
for our IR /NIR Microspectroscopy and Chemical Imaging investigations of Soybean seeds and Embryos. 


\section{FT-NIR Microscope: NIR AutoImage}

$\square \quad$ Introduced in 2002 by PerkinElmer Co. (Shelton, CT, USA) for high-resolution studies

$\square$ Employed for our NIR Microspectroscopy and Chemical Imaging investigations of Soybean seeds and Embryos

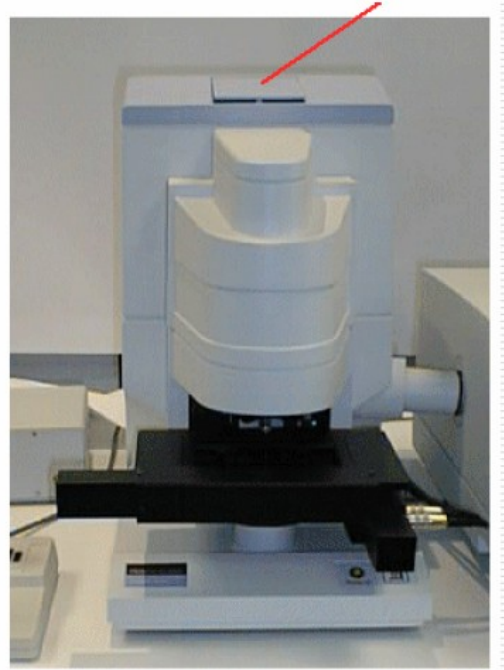




\section{AutoImage NIR Microscope Simplified Diagram for Operation in the Reflection Mode}

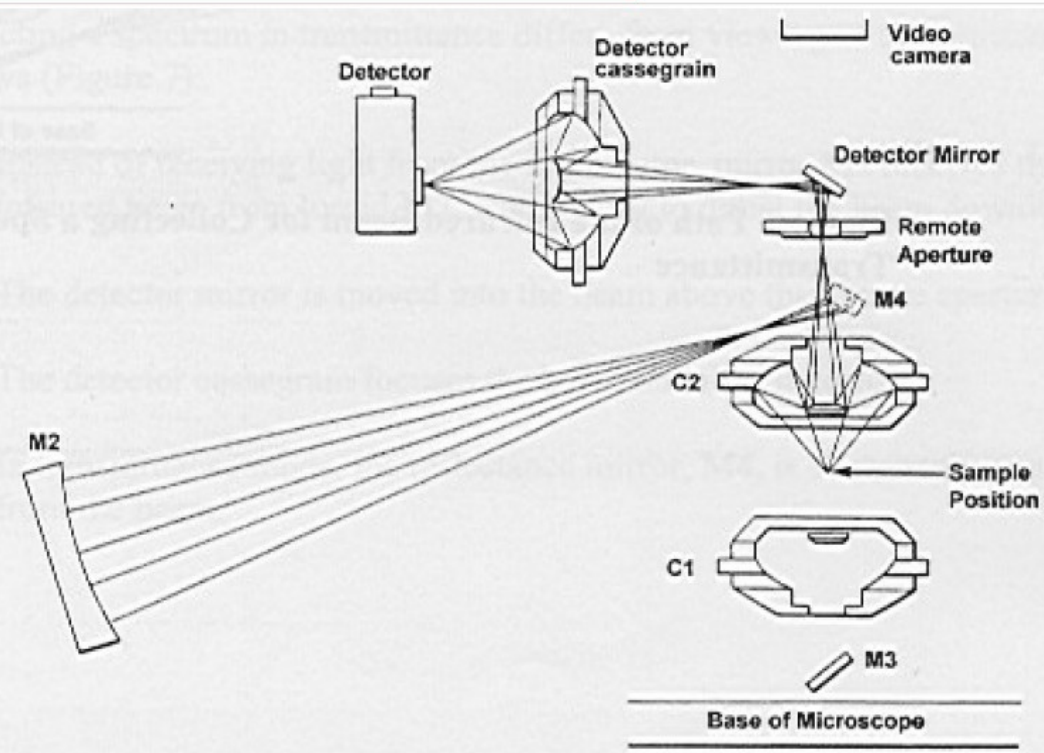




\section{FT-IR Microscope: Spotlight300}

$\square \quad$ Introduced in 2002 by PerkinElmer Co. (Shelton, CT, USA) for high-resolution studies

$\square$ Employed for our Mid-IR Microspectroscopy and Chemical Imaging investigations of thin sections of Soybean seeds and Embryos

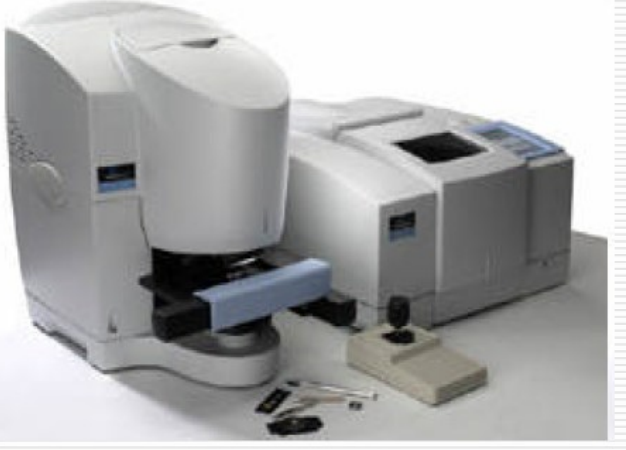


Figure 1A. FT-NIR Chemical Image of a Standard Sample Particle of 1 Micron Diameter Obtained with the AutoImage NIR Microscope in the Reflection Mode

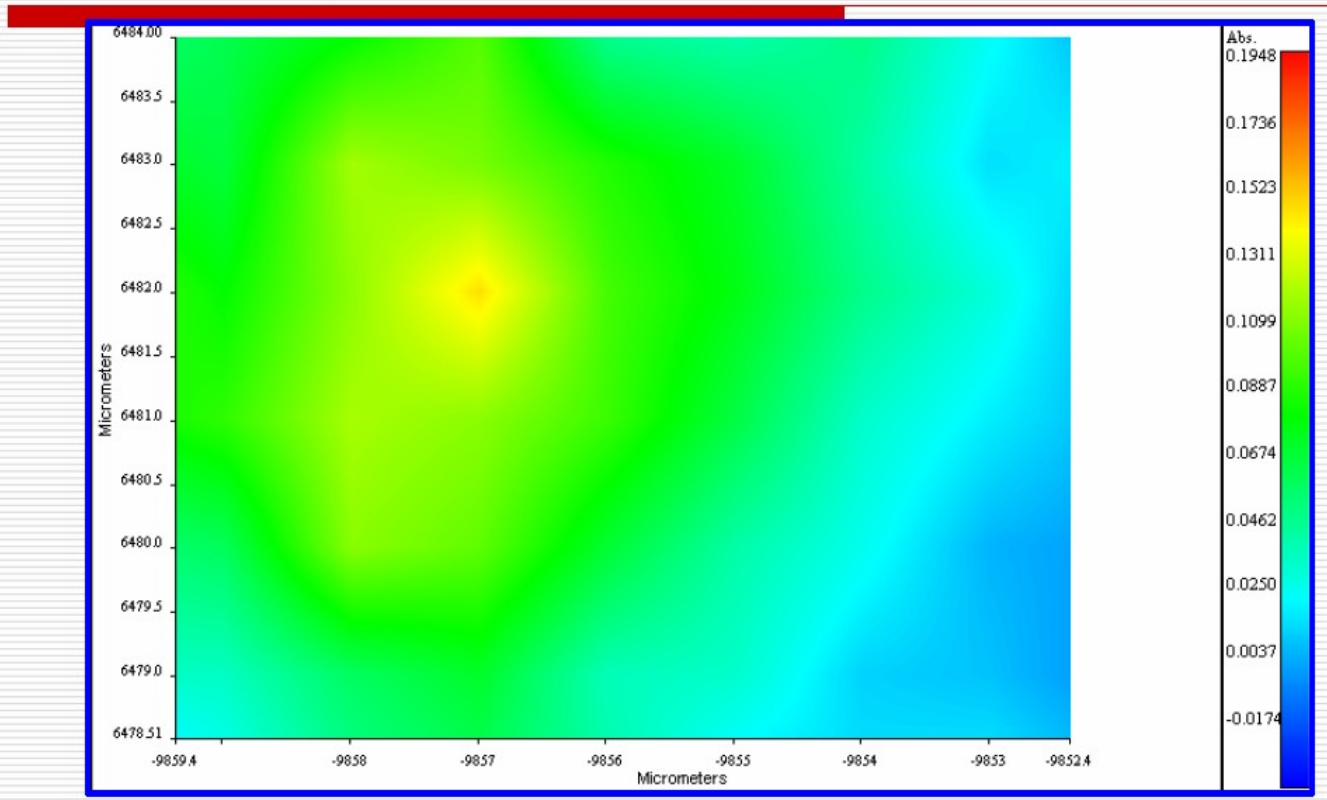


Figure 1B. Cross-section View of the FT-NIR Chemical Image of the 1 Micron Diameter Particle

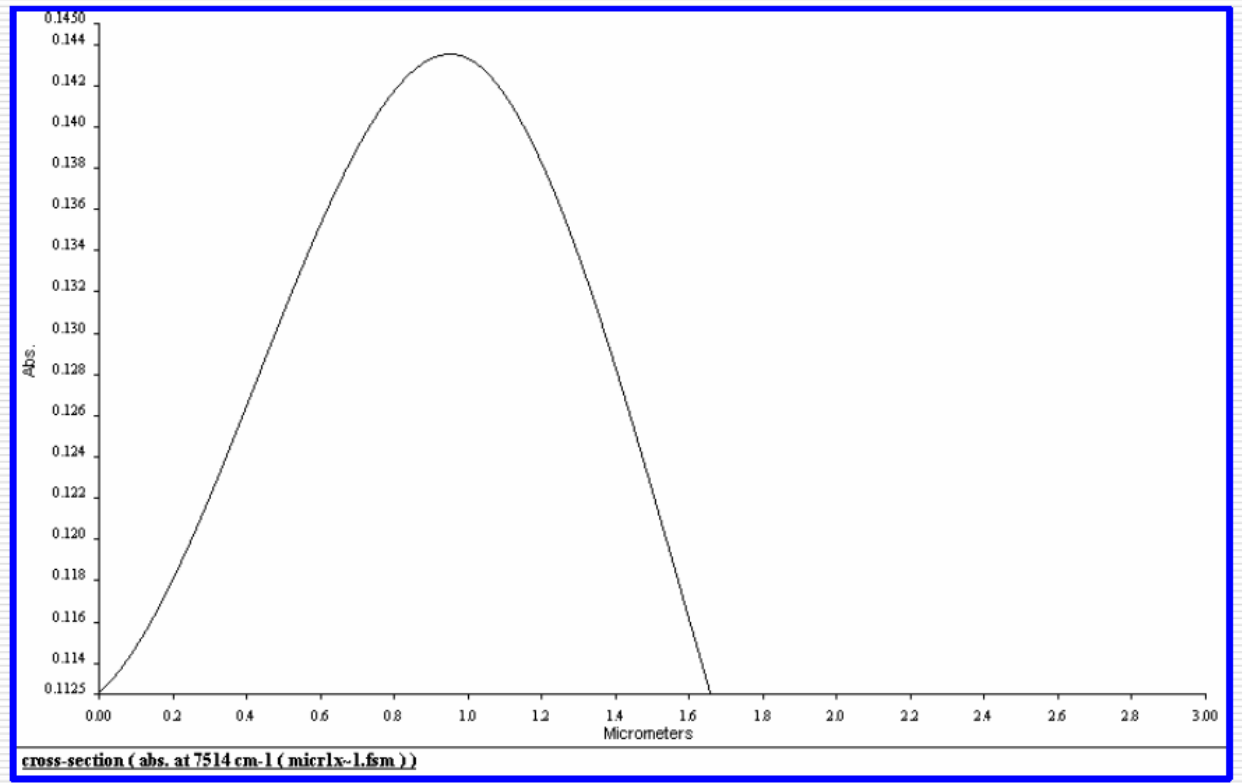


Figure 2A. NIR Image of Top Zone in a Cubic Zirconium Single Crystal at 1 Micron Resolution (Plot of the band ratio: $7211,5253 \mathrm{~cm}^{-1}$ )

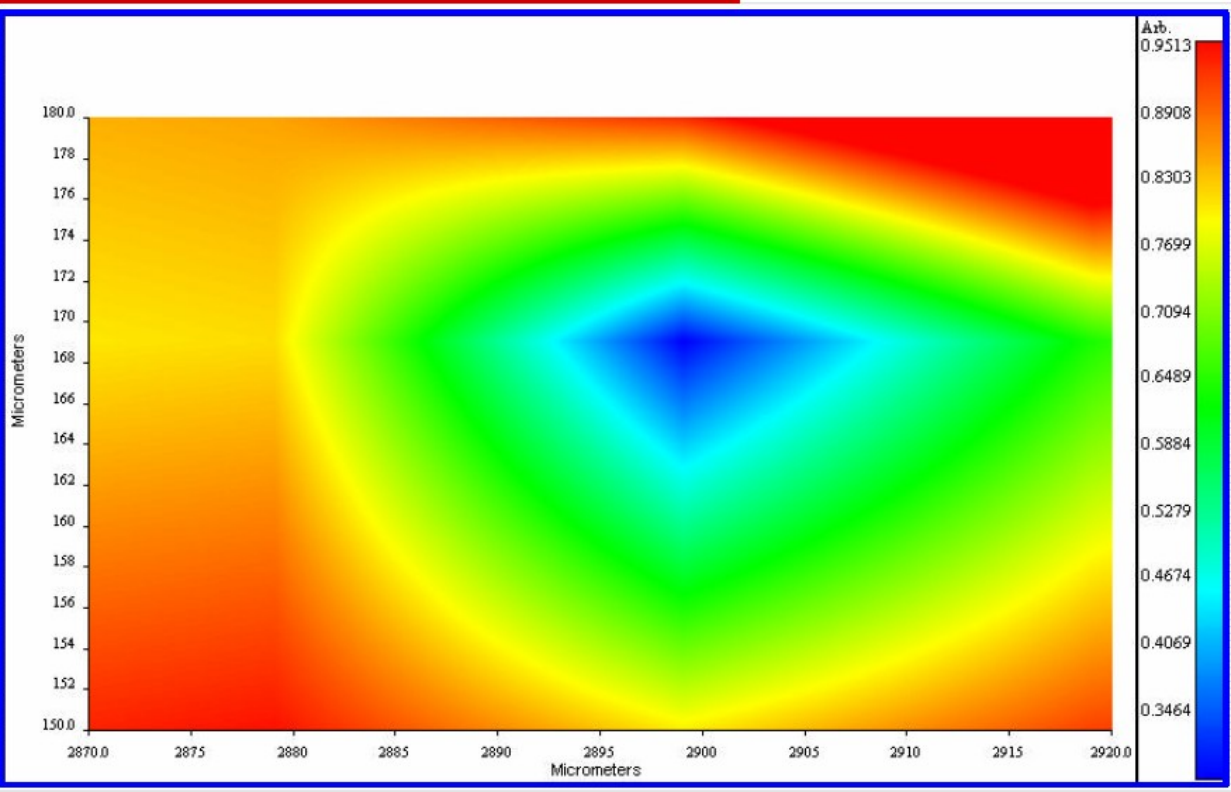


Figure 2B. NIR Image of a Cubic Zirconium Single Crystal at a Resolution of 1 Micron (Plot of the band ratio: $7253,5485 \mathrm{~cm}^{-1}$ )

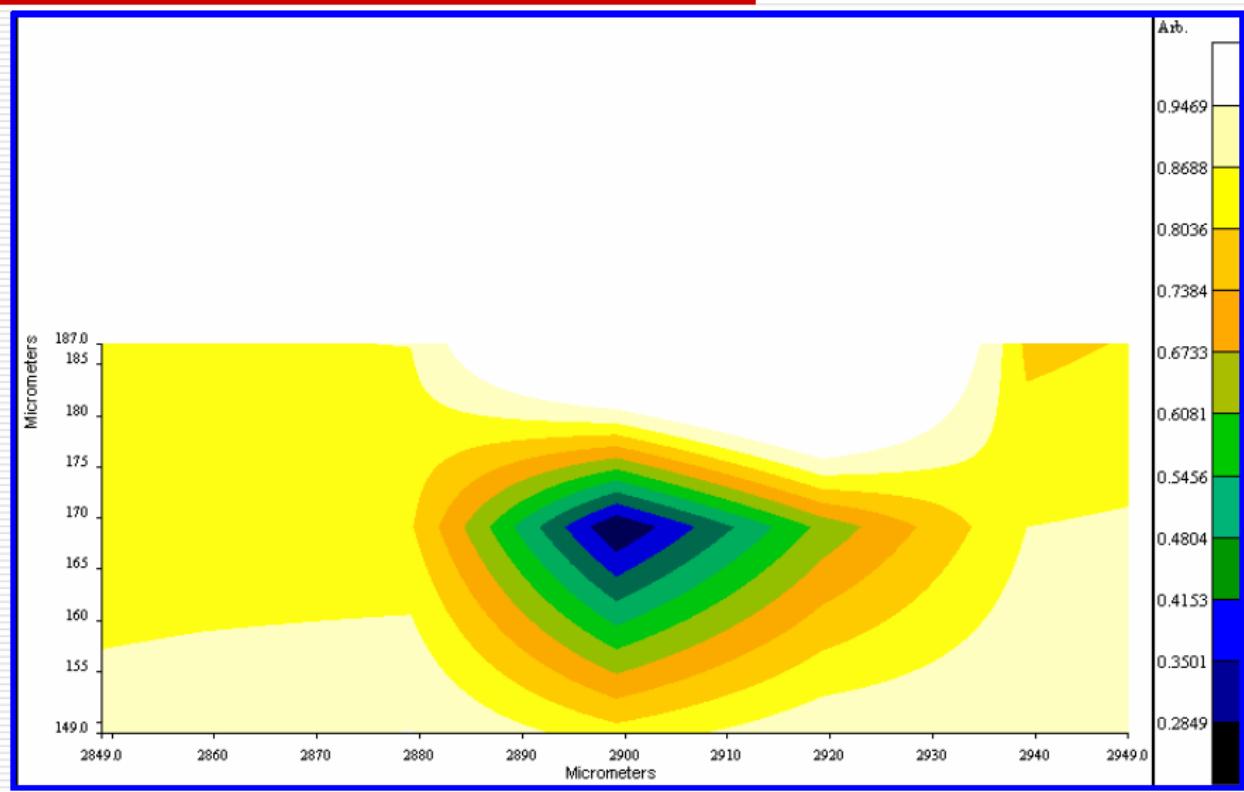


Figure 3. 3D Surface Project View of FT-IR Image of $10 \mu$ Diameter Spheres Obtained with the Spotlight 300 Microscope

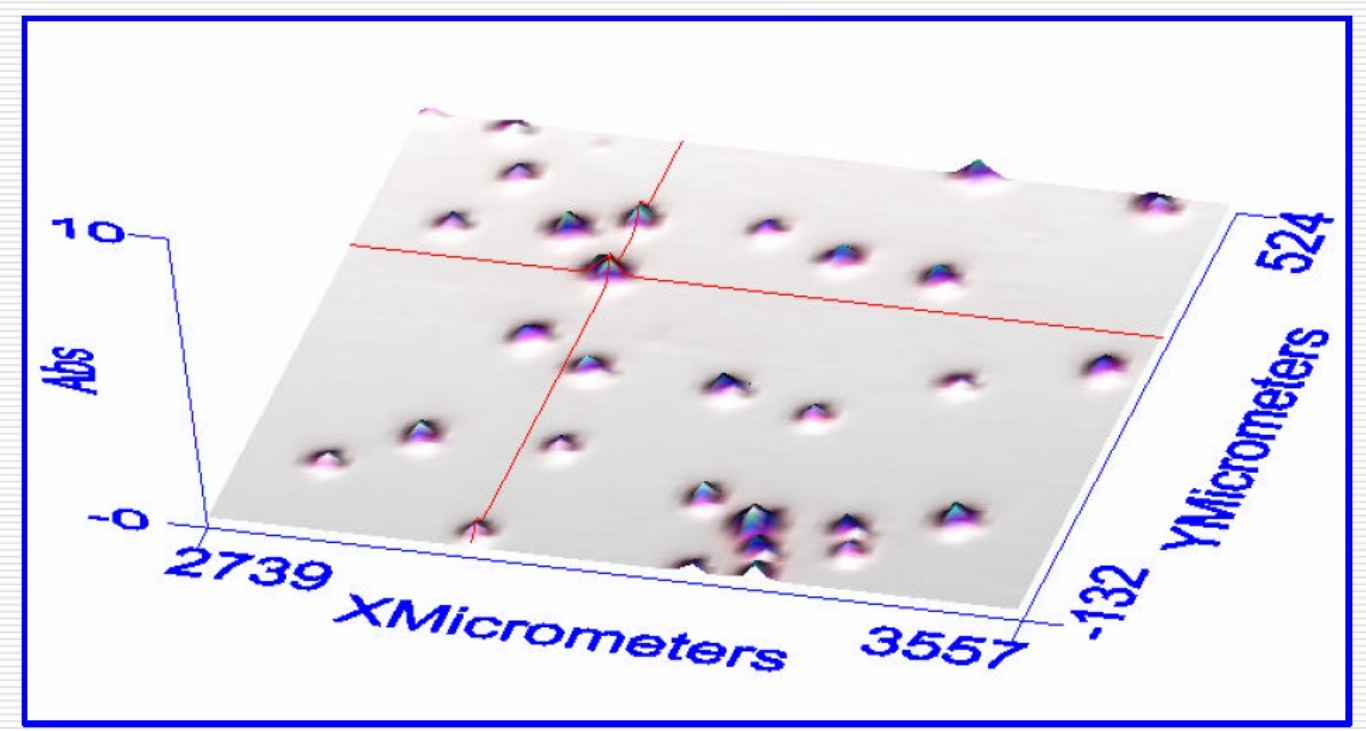


Figure 4. FT-IR Single Wavenumber Images $\left(761 \mathrm{~cm}^{-1}\right)$ of 6 Micron Diameter Latex Beads Obtained with the Spotlight 300 Microscope

3D Representation
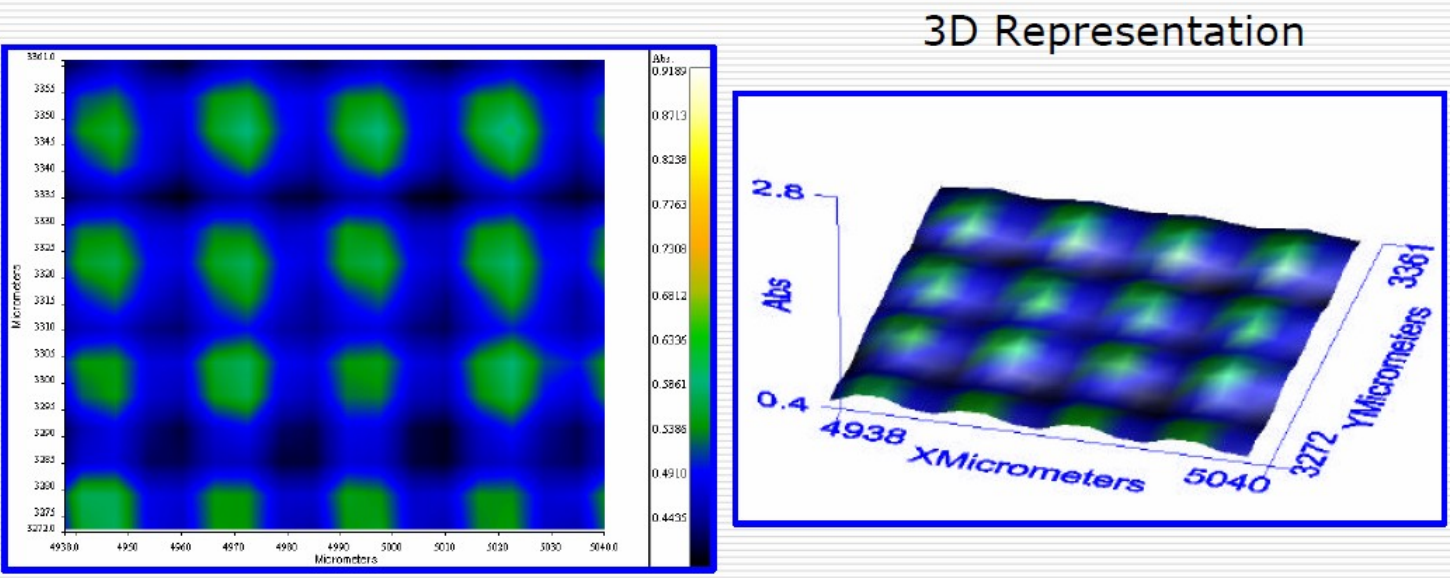
Figure 5. FT-IR Band Ratio Image

$\left(2157,961 \mathrm{~cm}^{-1}\right)$ of 6 Micron Diameter Latex Beads Obtained with the Spotlight 300

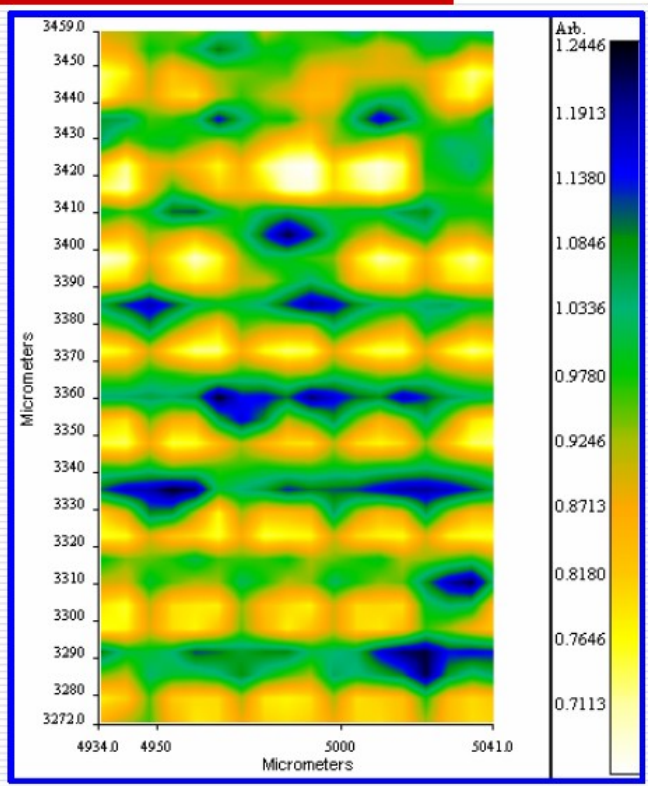


Figure 6. FT-IR Chemical Image (Left) and Visible Light Micrograph (Right) of a Black Coat Soybean with Part of the Coat Removed

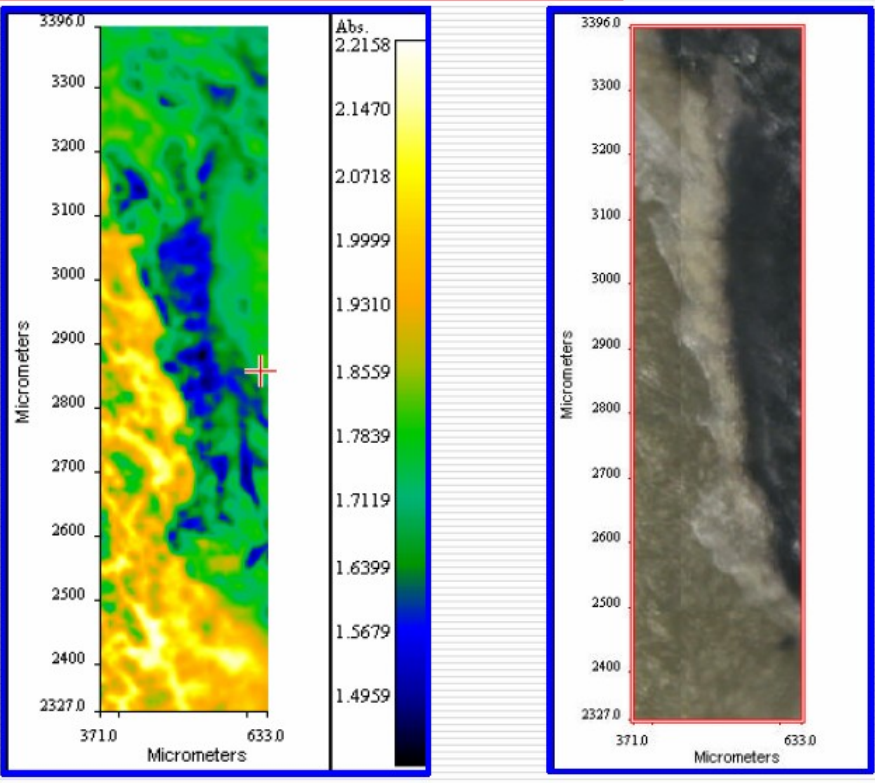




\section{Figure 7. Visible Image of a Soybean Embryo}

Sample at $9.6 \%$ initial moisture; 60X magnification.

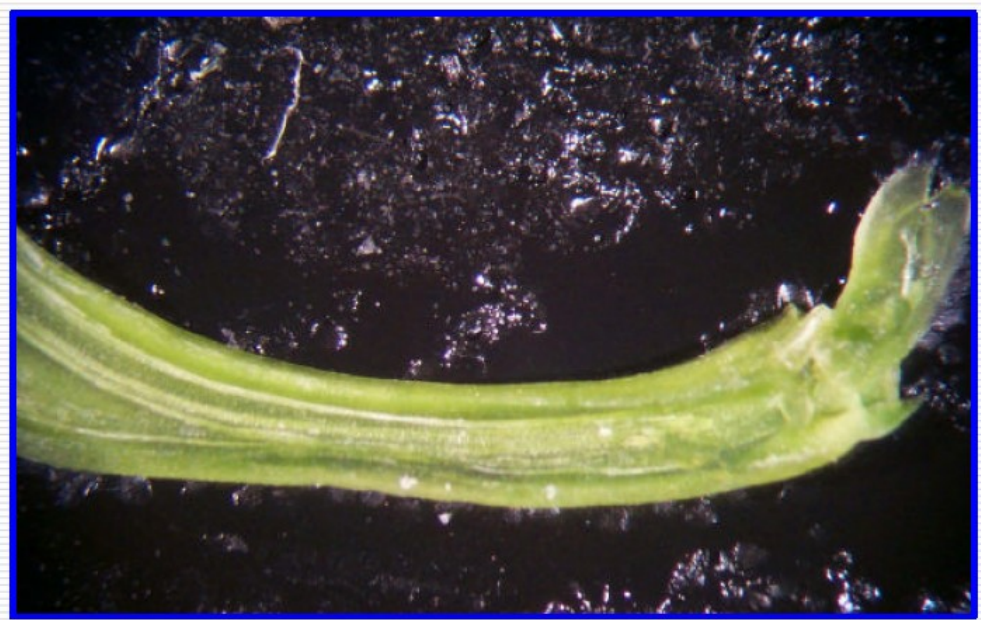


Figure 9. FT-IR Chemical Image of a Soybean Embryo (Amide Band Ratio)

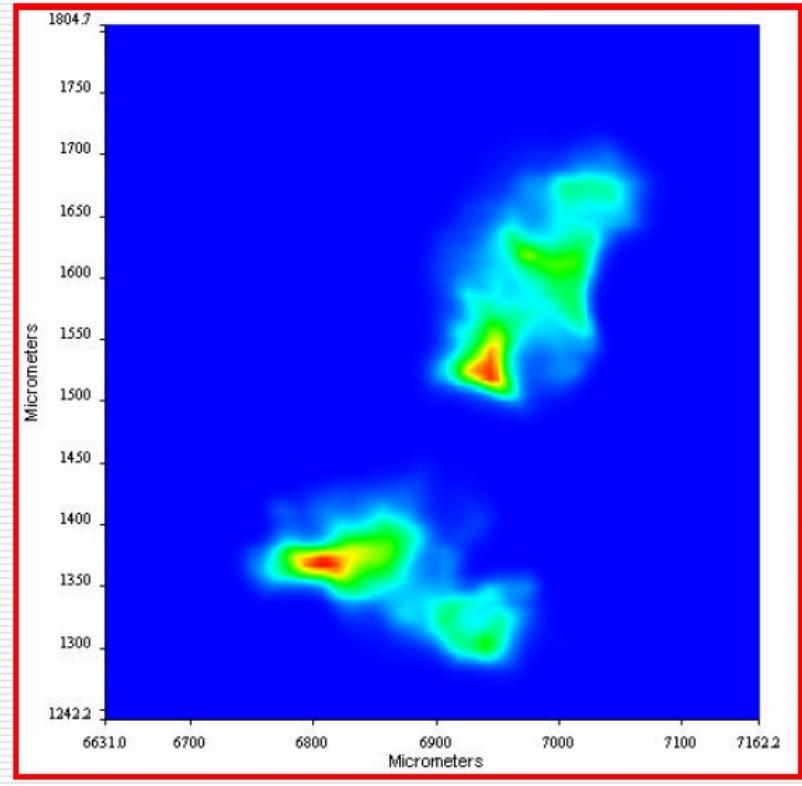


Figure 10. FT-NIR Chemical Image of Oil Distribution in a Mature Soybean Embryo Section

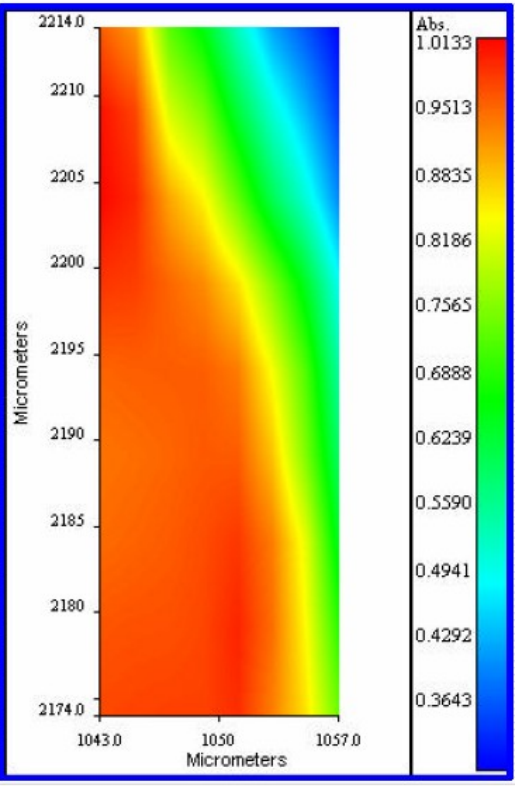


Figure 11. TEM Micrograph of a Suspension of Soybean Somatic Embryos in Culture (After treatment with $1 \mathrm{mM}$ Ethyl Methane Sulfonate)

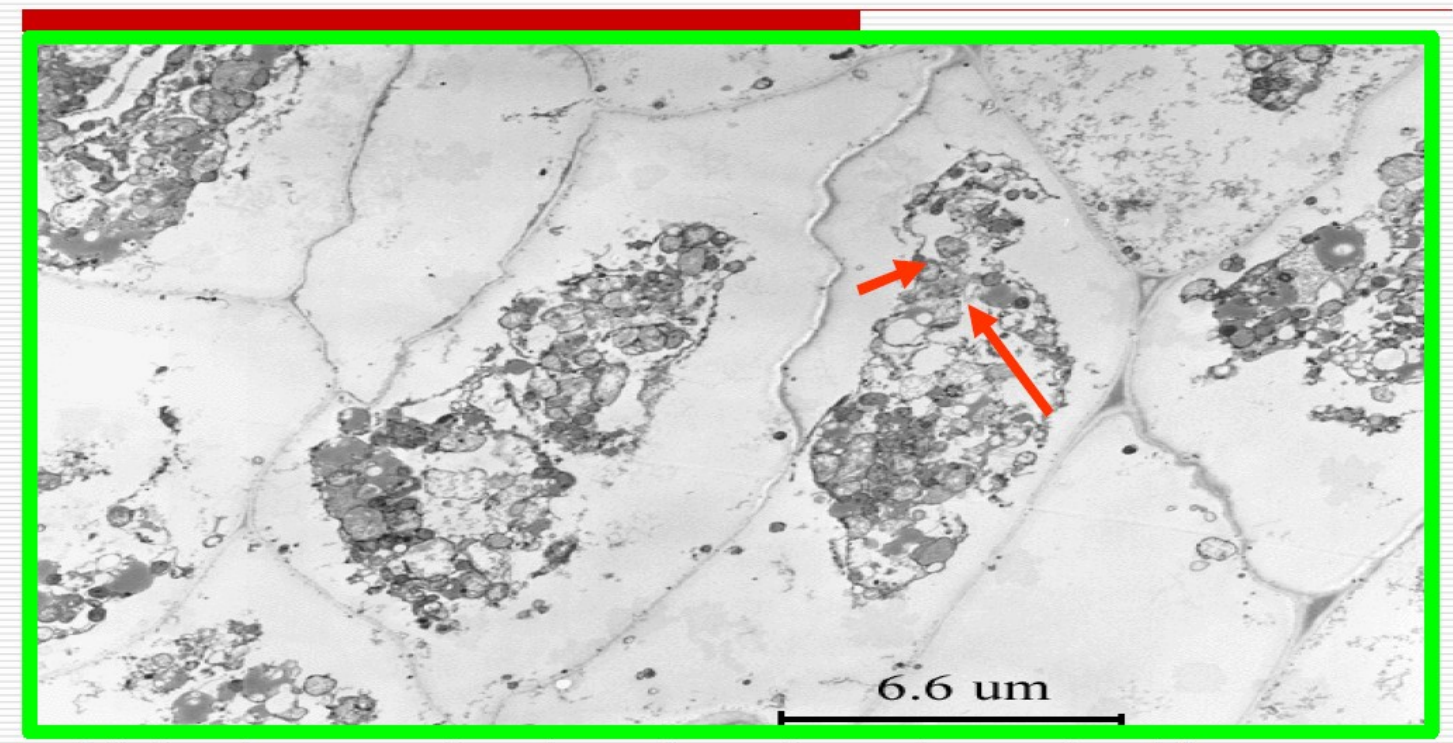

Oil droplets are distributed inside embryos (visible at arrows) in mutant soybean embryos with higher oil content. 
Figure 12. Proton NMR spectra of soybean oil showing direct proportionality of peaks area to the quantity of oil in the samples

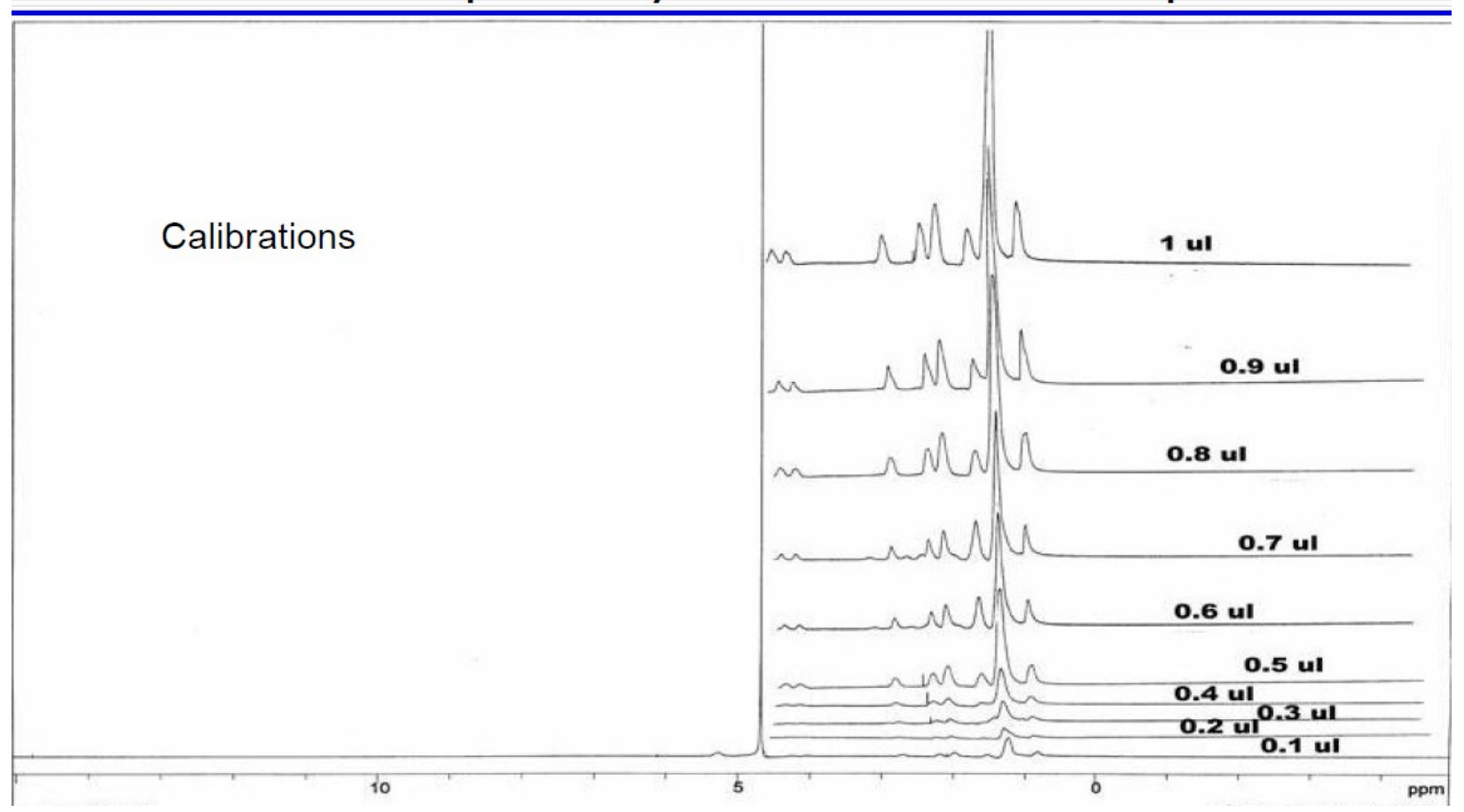


Figure 13. FT-NIR MicroImaging of 25-Micron MicroArrays

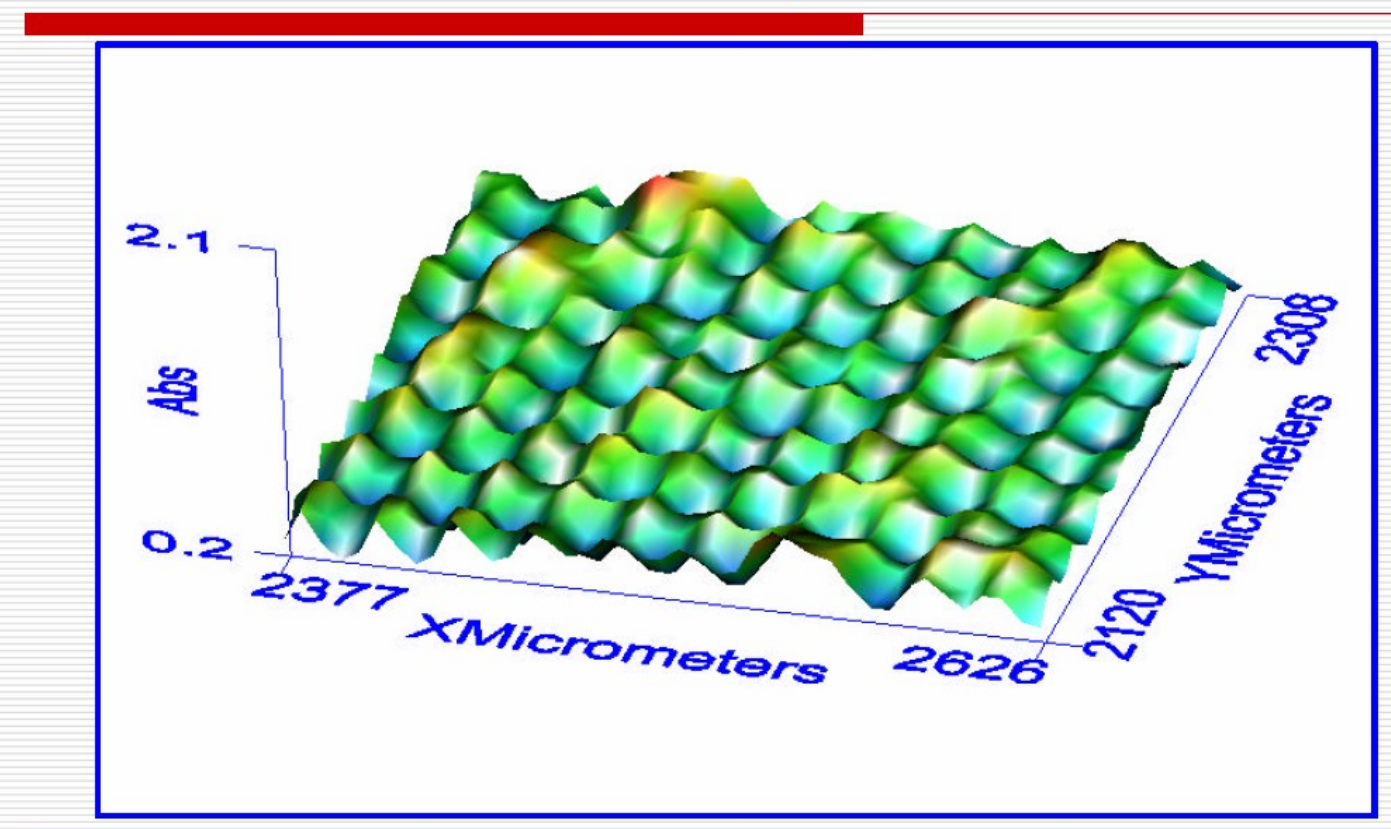


Figure 14. NIR Chemical Image of Human Tissue Cells in Culture following the Addition of Soy Isoflavones to Nutrients

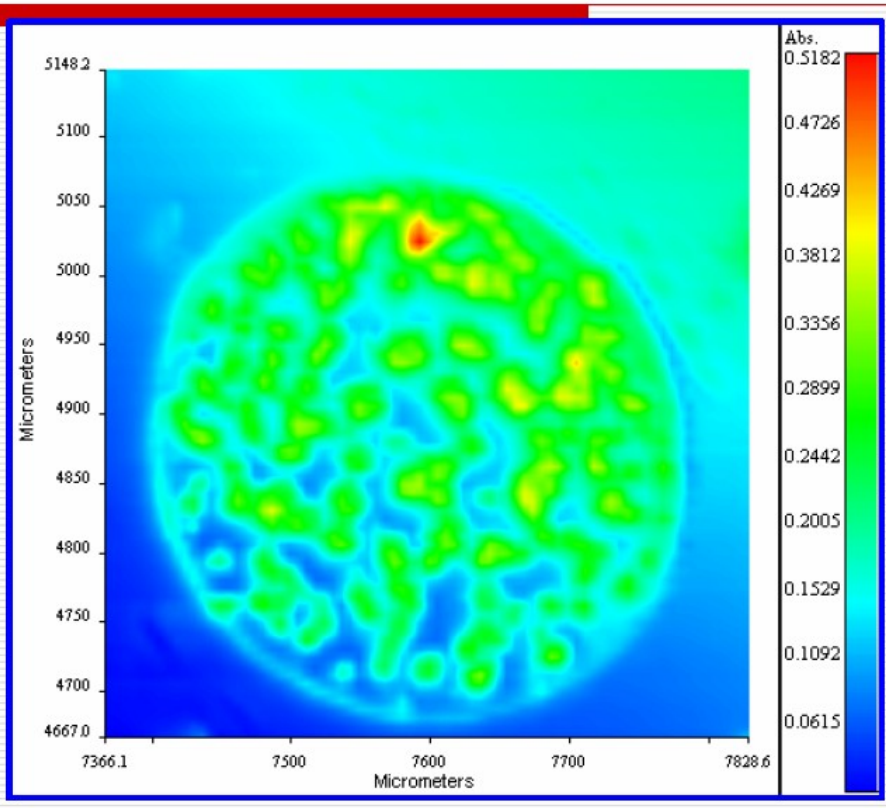


Figure 15. NIR Chemical Image and Visible Light Micrograph of Human Tumor Cells
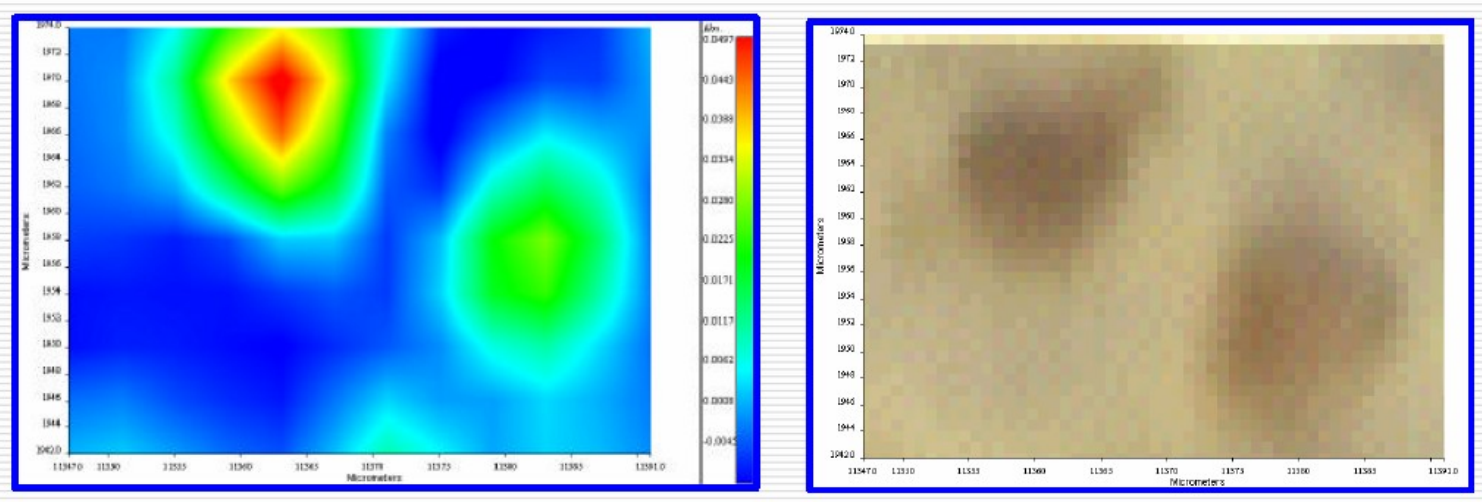


\section{Conclusions}

$\square$ Novel-design instrumentation for FT-IR/NIR Chemical Mapping, Microspectroscopy, and Fluorescence Microscopy is now capable of automated visualization of composition distribution and developmental changes in Single Soybean Seeds and Somatic Embryos with precision close to the picogram range. This may allow us to speed up the Genetic Selection of Soybean lines with Improved Oil or Protein, and Sugar composition.

High-resolution NIR Chemical Imaging may also enable rapid and sensitive analyses with micro-arrays for nucleic acids, multiple molecular bioassays, and automated proteomics that can help develop new Biotechnology, Biomedical \&

Pharmaceutical applications (ref. 2), such as those aimed at Cancer Prevention and its early Detection.

$\square$ Detailed nutritional studies in Cell cultures with phytochemicals, such as Soy Isoflavones (ref. 1), are now also made possible by FT-NIR Chemical Imaging of Single Cells either in culture or in vivo. 


\section{References Cited}

1. Tiefeng You, Guo, J., I. C. Baianu and Randall L. Nelson. "Determination of Isoflavone Contents for Selected Soybean Lines by FT-NIR Reflectance Spectroscopy." Soy2002 (Poster \# 505).

2. F.C. Clarke, Jee, D. R., Moffat, A. C. and Hammond, S.V. 2001. "Effective sample volume for measurements by near-infrared microscopy.".(e.g.: 40,000 um3, or 30 ng; Abstract, 116), British Pharmaceutical Conference. 